\title{
Entrevista a un profesional sanitario: estudio piloto de una actividad de contacto asistencial temprano en el grado en Medicina durante la pandemia por COVID-19
}

\author{
Natalia Jimeno-Bulnes, Álvaro Díez-Revuelta
}

Introducción. El Espacio Europeo de Educación Superior fomenta el diseño y la aplicación de metodologías docentes innovadoras, que en las ciencias de la salud pueden contribuir al conocimiento y el contacto temprano con la práctica asistencial, y a la humanización de la medicina. El objetivo es presentar las características y los resultados obtenidos en la actividad grupal 'Entrevista a un profesional sanitario'.

Sujetos y métodos. La actividad se ha desarrollado en la asignatura de Psicología, de segundo curso del grado en Medicina, durante el curso académico 2019-2020 ( $n=164$ estudiantes; 29,3\% hombres; edad media = 19,7 años). La pandemia por COVID-19 requirió su adaptación a la docencia no presencial. De los 33 profesionales sanitarios colaboradores, 29 contestaron al cuestionario de satisfacción en línea (65,5\% hombres; edad media = 49,2 años).

Resultados. Se constituyeron 33 grupos de estudiantes y la media (desviación estándar) de la calificación fue 8,8 $(0,65)$ sobre 10 puntos. Los estudiantes presentaron una amplia variedad de cuestiones relacionadas con la humanización de la medicina. Las entrevistas se realizaron fundamentalmente por videoconferencia (58,6\%). Los profesionales sanitarios que contestaron al cuestionario fueron preferentemente titulados en Medicina $(89,7 \%)$ y especialistas en medicina familiar y comunitaria (27,6\%). Presentaron una elevada satisfacción tanto en la organización de la actividad como en su valoración -media sobre 5 puntos (desviación estándar) , 4,5 (0,5) y 4,7 (0,3), respectivamente-.

Conclusión. 'Entrevista a un profesional sanitario' es una actividad grupal que facilita el contacto asistencial temprano y el papel activo de los estudiantes, ha sido muy bien valorada por los profesionales sanitarios participantes y puede adaptarse a la docencia no presencial.

Palabras clave. Educación a distancia. Educación médica de pregrado. Entrevista. Método docente. Profesional sanitario. Psicología médica.

Interviewing a health care professional: pilot study of an early care contact activity in the Medical degree during the COVID-19 pandemic

Introduction. The European Higher Education Area encourages the design and application of innovative teaching methods. Regarding Health Sciences, they can contribute to the early knowledge and contact with healthcare practice and to the humanization of medicine. The aim is to present the characteristics and results obtained in the group activity: 'Interview a healthcare professional'.

Subjects and methods. The activity has been applied in the subject of Psychology (second year of the Degree in Medicine) during the academic year 2019-20 ( $n=164$ students, $29.3 \%$ men, mean age $=19.7$ ). The COVID-19 pandemic has required its adaptation to distance education. Of the 33 collaborating health professionals, 29 answered the online satisfaction questionnaire $(65.5 \%$ men, mean age $=49.2$ years $)$.

Results. Thirty-three groups of students were constituted, the mean (standard deviation) rating was 8.80 (0.65) out of 10 points. Students presented a wide variety of issues related to the humanization of medicine. Most of the interviews were conducted by videoconference (58.6\%). The health professionals who answered the questionnaire were mostly graduates in Medicine (89.7\%) and specialists in Family and Community Medicine (27.6\%). They presented high satisfaction in both the organization and assessment of the activity -mean score out of 5 (SD), 4.5 (0.5) and 4.7 (0.3) respectively-.

Conclusion. 'Interview a healthcare professional' is a group activity that facilitates early contact with healthcare practice and the active role of students, it has been highly valued by the participating healthcare professionals and can be adapted to the non-face-to-face teaching modality.

Key words. Distance education. Health personnel. Interview. Medical psychology. Teaching method. Undergraduate medical education.
Área de Psiquiatría. Facultad de Medicina. Universidad de Valladolid. Valladolid, España.

Correspondencia: Dra. Natalia Jimeno Bulnes. Área de Psiquiatría. Facultad de Medicina. Edificio de Ciencias de la Salud. Av. Ramón y Cajal, 7. E-47005 Valladolid.

E-mail:

natalia.jimeno@uva.es

Agradecimientos:

A todos los profesionales sanitarios que se han ofrecido a participar en la actividad, por su disponibilidad; al Colegio Oficial de Médicos de Valladolid, por su desinteresada colaboración, y a los estudiantes de la asignatura, porque sin su esfuerzo continuado esta actividad no tendría sentido.

Recibido:

02.02.21.

Aceptado: 27.05.21.

Conflicto de intereses: No declarado.

Competing interests: None declared.

Cómo citar este artículo: Jimeno-Bulnes N, Díez Á. Entrevista a un profesional sanitario: estudio piloto de una actividad de contacto asistencial temprano en el grado en Medicina durante la pandemia por COVID-19. FEM 2021; 24: 199-209. doi: 10.33588/ fem.244.1136.

(c) $2021 \mathrm{FEM}$ 


\section{Introducción}

En España, el diseño del programa de estudios de cada grado universitario se fundamenta en el correspondiente 'libro blanco' o documento oficial que, entre otros aspectos, clasifica y establece las diversas competencias que deben alcanzarse [1]. La formación basada en competencias constituye el medio adecuado para favorecer la formación de profesionales de ciencias de la salud y su continua adaptación a los retos sociosanitarios actuales, lo cual supone la modificación del modelo educativo actual [2].

La enseñanza y el aprendizaje de estas competencias requieren, indudablemente, la aplicación de una diversidad de métodos docentes, que idealmente deben ser objeto de investigación, desarrollo e innovación [3]. Ejemplos de ello son el uso de maquetas [4], herramientas tecnológicas como los clickers y Kahoot [5], en ambos casos en estudiantes de ciencias de la salud de Chile, o la asignatura Introducción al sistema de salud, propuesta en la Universidad de Barcelona, en la que se presentan conceptos diversos con el fin de integrar mejor los conocimientos básicos y clínicos correspondientes a los cursos siguientes [6]. Se ha sugerido, además, que el contacto precoz de la actividad asistencial, habitualmente por medio del inicio temprano de prácticas hospitalarias, facilita la motivación y el aprendizaje del estudiante de Medicina [7].

La asignatura Psicología médica, incluida en el bloque 2, Patología humana, debe facilitar al estudiante, entre otros contenidos, conocer las bases psicosociales del enfermar humano y los conceptos generales de la medicina psicosomática [1]. Se dirige, pues, a competencias $(\mathrm{C})$ como: 'reconocer los determinantes de salud en la población', incluidos los psicológicos y sociales (C25), 'reconocer las bases de la conducta humana normal y sus alteraciones' (C08) y 'escuchar con atención, obtener y sintetizar información pertinente acerca de los problemas que aquejan al enfermo y comprender el contenido de esta información' (C21) [1]. La aplicación de principios psicológicos es un elemento esencial en la formación del médico en el siglo XXI [2]. Incluso se ha propuesto la realización de una historia clínica médico-psicológica, por parte de estudiantes que inician su formación clínica, como estrategia que permite el contacto directo con el ser humano enfermo y no solamente con la enfermedad [8]. Por otra parte, son elementos esenciales de la humanización de la medicina tanto la individualidad del paciente como la excelencia y el humanismo (valores humanos y profesionales) del médico [9].
Con la finalidad de contribuir a este conocimiento y contacto temprano con la práctica asistencial, fomentar la humanización de la medicina y también incrementar la motivación de los estudiantes de segundo curso del grado en Medicina en la asignatura de Psicología, se estableció durante el curso académico 2019-2020 una actividad docente innovadora: 'Entrevista a un profesional sanitario'. A pesar de que la actividad no se había diseñado para una situación de confinamiento, el escenario de pandemia por COVID-19 requirió, al menos en la segunda mitad del curso, una aplicación no presencial de la actividad. Nuestros objetivos en el presente estudio son: a) presentar las características y la implementación de dicha actividad durante el mencionado curso académico, en el marco de una docencia no presencial debida a la situación de pandemia por COVID-19, y b) exponer las características principales de los estudiantes y profesionales sanitarios participantes, así como los resultados iniciales obtenidos en la actividad y su valoración.

\section{Sujetos y métodos}

Se trata de un estudio observacional y transversal, realizado tras la aplicación de la actividad propuesta en la asignatura de Psicología del grado en Medicina de la Universidad de Valladolid. Dicha asignatura tiene un total de cuatro créditos ECTS, está actualmente a cargo de dos profesores (médico psiquiatra y psicólogo) y se imparte habitualmente a unos 160 estudiantes en el segundo cuatrimestre del curso académico (febrero a junio) de forma presencial.

La actividad 'Entrevista a un profesional sanitario' tiene como objetivos fundamentales contribuir al conocimiento y contacto temprano con la práctica asistencial y a la humanización de la medicina por medio de la experiencia de un profesional sanitario experimentado. Ha sido diseñada por los dos profesores de la asignatura para su inicio en el curso académico 2019-2020 y corresponde a la práctica 'Aspectos psicológicos de la práctica clínica'. La actividad consiste en la realización por parte de grupos de cuatro a seis estudiantes de una entrevista a un profesional sanitario -que voluntariamente ha accedido a ser entrevistado- acerca de los contenidos de interés para la práctica clínica que han sido previamente impartidos en la asignatura de Psicología del grado en Medicina. Estos contenidos incluyen seis temas teóricos: 'Afectividad y entrevista médicopsicológica, 'Psicología de la salud y la enfermedad', 'Psicología del enfermo en situaciones específicas', 'El estrés,' ‘Factores del proceso curativo y psicotera- 
pia' e 'Intervención en crisis'. La duración recomendable de la entrevista es de una hora. La calificación en la actividad tuvo un peso de 1 punto sobre 10 en la calificación final de la asignatura.

Si bien el inicio del período de docencia no virtual el día 14 de marzo de 2020, debido a la pandemia por COVID-19, requirió la modificación de algunos aspectos, la organización e implementación de la actividad se establecieron en una serie de etapas y plazos de la forma siguiente (Figura):

- Julio de 2019 a enero de 2020: contacto y convenio de colaboración con el Colegio Oficial de Médicos de Valladolid (COMVA). Se contó con el apoyo inmediato del Decanato de la Facultad de Medicina de la Universidad de Valladolid y la inestimable ayuda del COMVA, con el que se contactó inicialmente en julio de 2019. Todo ello se formalizó en un convenio de colaboración docente ad hoc entre ambas instituciones, firmado el 20 de enero de 2020. La colaboración del COMVA consistió en difundir, a los colegiados con actividad clínica superior a cinco años, un mensaje con el folleto informativo en el que se presentaba brevemente la actividad, con el contacto de los organizadores de la actividad por medio de una cuenta de correo electrónico específica abierta con esta finalidad.

- Enero y febrero de 2020: constitución de una red de profesionales sanitarios voluntarios. Igualmente se difundió la actividad a otros contactos del profesorado. Así pues, se propuso la actividad fundamentalmente, pero no de forma exclusiva, a médicos de distintas especialidades y ámbitos. Éstos debían remitir cumplimentado un sencillo formulario adjunto al folleto informativo que recogía sus principales datos profesionales y de contacto: nombre, correo electrónico, profesión, especialidad, años de experiencia, centro de trabajo y posible autorización a grabar la entrevista en formato audio o audio-vídeo. Los interesados podrían remitir su información hasta el comienzo de la docencia de la asignatura, el 10 de febrero de 2020.

- Febrero de 2020: exposición breve a los estudiantes de las características generales de la actividad. Se realizó en la presentación de la asignatura el día 10 de febrero de 2020.

- Febrero y marzo de 2020: constitución de los grupos de trabajo. Éstos fueron de cuatro a seis estudiantes, a elección voluntaria, los mismos para todas las actividades grupales de la asignatura. Cada grupo debía contar con un/a coordinador/a, que representaba el contacto entre el grupo y el profesorado. Dado el retraso
Figura. Diagrama de Gantt con las etapas, cronograma y plazos establecidos de la actividad 'Entrevista a un profesional sanitario' durante el curso académico 2019-2020.

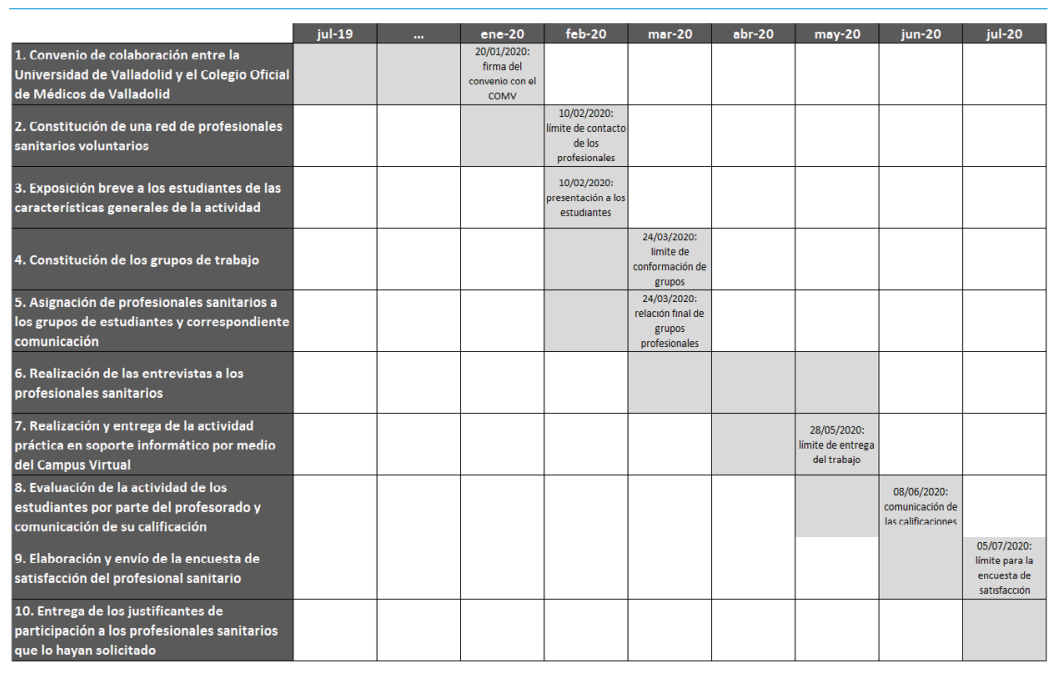

de parte de los estudiantes en constituir estos grupos, en primer lugar, así como el inicio del período de docencia no presencial como consecuencia del inicio del estado de confinamiento, fue preciso extender el plazo inicial del 21 de febrero de 2020 al 24 de marzo de 2020.

- Febrero y marzo de 2020: asignación de profesionales sanitarios a los grupos de estudiantes y correspondiente comunicación. El hecho de que el número de profesionales voluntarios fuera muy ajustado al número previsible de grupos de estudiantes y el retraso en la constitución de éstos -como se ha indicado- motivó que se estimulara a los estudiantes a buscar entre sus posibles contactos, en lo posible, a un profesional sanitario que accediera a participar en la actividad, para lo cual debían remitir a la coordinadora el mencionado formulario debidamente cumplimentado. El 24 de marzo de 2020 se presentó a los estudiantes la relación final de profesionales sanitarios voluntarios para la actividad, en concreto la titulación (Medicina, Enfermería, etc.), especialidad y centro de trabajo, para que procedieran a elegir a un profesional sanitario. Se procedió a continuación a enviar un mensaje a cada uno de los profesionales participantes, comunicándoles, en su caso, la asignación del grupo de estudiantes, con copia al coordinador/a del grupo, con el fin de facilitar el contacto entre ambos para la realización de la actividad. Para facilitar la gestión de información, esta tarea 
fue asumida por la profesora coordinadora de la actividad (N.J.B.).

- Marzo a mayo de 2020: realización de las entrevistas a los profesionales sanitarios. Una vez realizada la asignación del profesional en la mayor parte de los grupos, el 6 de marzo de 2020 - por tanto, todavía en período de docencia presencial- se publicó un documento de información a estudiantes acerca de la actividad con las correspondientes normas de realización, calendario previsto y evaluación. Entre otros aspectos se incluyeron: la disponibilidad de la Facultad de Medicina de la Universidad de Valladolid y el COMVA para la realización de las entrevistas; los contenidos y duración de la entrevista; las características del documento de texto que debían realizar a continuación; el modo de entrega, y los criterios de evaluación de la actividad. Aunque previamente se había establecido que debían ser presenciales y directas, debido a la situación sanitaria en nuestro entorno en la fecha de publicación de las normas referidas y el inicio del período de docencia no presencial en los días siguientes (13 de marzo de 2020), se autorizó su realización de forma telemática o por cualquier otro medio.

- Abril y mayo de 2020: realización y entrega de la actividad práctica en soporte informático por medio del campus virtual. La entrega, con fecha límite el 28 de mayo de 2020, incluyó necesariamente un documento de texto, y de forma adicional, en los casos autorizados por el profesional, y recogido por los estudiantes, un archivo de audio o audio-vídeo.

- Mayo y junio de 2020: evaluación de la actividad de los estudiantes por parte del profesorado y comunicación de su calificación. Para ello se emplearon los criterios previamente establecidos y que se desarrollaron en el documento de evaluación de la actividad: calidad de las preguntas formuladas (40\%), calidad del comentario o reflexión acerca de algunos contenidos de la entrevista (40\%) y aspectos formales (20\%). Este documento y las calificaciones obtenidas por los grupos de estudiantes se publicaron el 8 de junio de 2020.

- Junio y julio de 2020: elaboración y envío de la encuesta de satisfacción del profesional sanitario. Se utilizó para ello un formato telemático por medio de Google Forms ${ }^{\odot}$. La encuesta de satisfacción del profesional sanitario incluyó los siguientes contenidos: dirección de correo electrónico, sexo, edad, titulación profesional, especialidad, centro actual de trabajo, universidad en la que cursó la titulación, año de obtención de la titulación, año de obtención de la especialidad principal, forma en que obtuvo conocimiento de la actividad, modo de realización y contestación de la entrevista, y preferencia de realización de la actividad en caso de no confinamiento. Asimismo, incluyó una serie de escalas de tipo Likert ( 1 a 5 , desde 'muy en desacuerdo/mal/insatisfecho' hasta 'muy de acuerdo/bien/satisfecho') para la valoración de la organización (información recibida, proceso de inscripción, asignación de estudiante y realización de la entrevista) y de la calidad de la actividad por parte de los estudiantes (tiempos empleados, adecuación de las preguntas, utilidad formativa y satisfacción general). En el formulario también se preguntó a los profesionales si deseaban recibir un justificante de participación y retroalimentación de la actividad. El mensaje incluyó el agradecimiento expreso por su participación y la valoración positiva de la experiencia referida por muchos estudiantes. Se estableció una fecha límite de entrega de la encuesta para el 5 de julio de 2020.

- Julio de 2020: entrega de los justificantes de participación a los profesionales sanitarios que lo hubieran solicitado.

\section{Resultados}

\section{Resultados de la entrevista}

A partir de las indicaciones establecidas, cada uno de los 33 grupos de estudiantes ha elaborado previamente un conjunto de, al menos, 10 preguntas para la entrevista al correspondiente profesional sanitario, preguntas que eventualmente se han modificado durante el desarrollo de la misma. En la tabla I se presenta un modelo de entrevista al profesional sanitario con preguntas clave acerca de los temas principales de la asignatura para la actividad propuesta (Tabla I). Respecto a las manifestaciones de los profesionales sanitarios, se han recogido muy diversas aportaciones, entre las que, a modo de ejemplo, se han seleccionado las recogidas en la tabla II.

\section{Características de los participantes y de la aplicación de la actividad propuesta}

El número de estudiantes matriculados en la asignatura en el curso fue 164 (edad media $=19,7$ años; 48 hombres y 116 mujeres), distribuidos, a 
Tabla I. Preguntas clave de la entrevista a un profesional sanitario que han sido seleccionadas o propuestas por los profesores organizadores de la actividad.

Tema 5. Afectividad y entrevista médico-psicológica

- ¿Considera que la empatía es una característica importante en el médico o profesional sanitario?

- En una entrevista clínica, ¿qué cualidades o características del paciente, o sus familiares, considera imprescindibles?

- En una entrevista clínica, ¿qué cualidades o ccaracterísticas del profesional clínico considera imprescindibles?

- ¿Le sucede que en su práctica clínica tiene que adaptar el lenguaje técnico a las características del paciente, por ejemplo, en una entrevista?

\section{Tema 12. Psicología de la salud y la enfermedad}

- ¿Cree que influyen los factores psicológicos o sociales en el mantenimiento de la salud? Por ejemplo, las características de la personalidad, el estado de ánimo, la relación de la persona con sus familiares o personas con quien convive, etc.

- Y ahora, respecto a la enfermedad, ¿cree que influyen los factores psicológicos o sociales en la aparición de la enfermedad?

- En su experiencia, ante el diagnóstico de una enfermedad, ¿existe una única respuesta ante la enfermedad? ¿Puede comentar un poco más al respecto?

- ¿Es frecuente que el paciente se oponga o rechace un tratamiento? Si le ha sucedido, ¿qué hace en ese caso?

- ¿Qué se espera de 'un buen paciente'? ¿Y de 'un buen médico o profesional sanitario'?

- ¿Ha observado alguna vez que una actuación del profesional sanitario ocasione algún tipo de daño al paciente? En su caso, ¿puede darnos más detalles?

Tema 13. Psicología del enfermo en situaciones específicas

- En general, ¿qué supone o cómo viven sus pacientes un ingreso hospitalario? ¿Y una intervención quirúrgica?

- En su experiencia, ¿cuáles son los principales factores que influyen en la respuesta psicológica de un paciente con cáncer? ¿Cuál es su respuesta más frecuente?

- Independientemente del tratamiento en sí, ¿cómo puede ayudar el médico, o bien otro profesional sanitario, a un paciente con cáncer? ¿Y a un enfermo terminal?

- ¿Cómo responde un paciente ante una enfermedad terminal? ¿Qué factores influyen en esta respuesta?

- ¿Hay algún caso que le haya impactado especialmente por estos aspectos psicológicos o sociales que estamos comentado?
Tema 14. Factores del proceso terapéutico

- ¿Cuáles cree que son las habilidades principales personales, más allá de las profesionales, que debe poseer un médico u otro profesional sanitario?

- ¿Qué aspectos personales del paciente debería explorar el profesional clínico más allá de la sintomatología clínica que causa la demanda?

- ¿Qué importancia le da al conocimiento del contexto físico y social del paciente?

- ¿Hasta qué punto cree que debe el paciente ser parte activa en la selección de recursos diagnósticos y terapéuticos?

- ¿Cómo piensa que se puede motivar e implicar al paciente de forma más activa en su terapéutica?

- ¿Qué consejos podría darnos para optimizar la relación con el paciente durante la entrevista y el proceso diagnóstico?

Tema 15. Intervención en crisis

- ¿Qué características presenta el paciente que requiere un abordaje urgente?

- ¿Cuáles cree que deberían ser los pasos que tendría que seguir el profesional clínico cuando un paciente no reconoce una conducta peligrosa y dañina para su salud?

- ¿Qué papel debería tener el médico en las situaciones de urgencia y primeros auxilios psicológicos entre los diversos profesionales clínicos?

- ¿Ha intervenido alguna vez en una situación en la que un paciente requiera primeros auxilios psicológicos urgentes? ¿Cómo actuó y cómo cree que podría mejorar su actuación en futuras situaciones similares?

- ¿Cómo procedería ante una situación que requiera primeros auxilios psicológicos (por ejemplo, ante un accidente o catástrofe)?

- ¿Cómo cree que debería comunicarse y relacionarse el profesional clínico con una persona que requiera abordaje psicológico urgente para evitar consecuencias potencialmente dañinas?

\section{Tema 16. El estrés}

- ¿Cree que el estrés puede influir en la aparición de una enfermedad? En su caso, ¿de qué forma?

- ¿Considera que el estrés puede influir en la evolución de una enfermedad y en la respuesta al tratamiento?

- En su experiencia, ¿pueden influir factores familiares, sociales o de otro tipo en el afrontamiento y el pronóstico de una enfermedad?

- ¿Existe algún factor o aspecto que le produzca estrés en su trabajo? En su caso, ¿puede indicarnos de qué tipo?

- ¿Cómo maneja su propio estrés, por ejemplo, durante el trabajo o fuera del mismo?

- ¿Cómo ha vivido el confinamiento debido a la pandemia por CO VID-19? ¿Y cómo está viviendo ahora la pandemia? su vez, en dos grupos de clase, con 83 y 81 estudiantes, respectivamente. La principal vía de acceso de los estudiantes al grado fue a través de las pruebas de acceso a la universidad ( $n=160$; $97,6 \%)$, mientras que dos estudiantes accedieron por reconocimiento de estudios y otros dos a través de programas internacionales $(1,2 \%$ en cada caso). La mayoría de los estudiantes cursaba la asignatura por primera vez $(n=156 ; 95,1 \%)$, siete estudiantes se encontraban en segunda matricu- lación $(4,3 \%)$ y un solo estudiante en cuarta matriculación (0,6\%) (Tabla III). El número de grupos constituidos para la actividad fue 33. Por su parte, el número total de profesionales sanitarios participantes fue igualmente 33, en su mayoría médicos de distintas especialidades y ámbitos. De los 33 profesionales, 29 contestaron a la encuesta de satisfacción (87,9\%; edad media: 49,2 años; 10 hombres y 19 mujeres). El número promedio de años desde que obtuvieron su titulación fue de 
Tabla II. Respuestas seleccionadas que han sido obtenidas en las entrevistas a profesionales sanitarios.

Tema 12. Psicología de la salud y la enfermedad

- Los médicos tenemos que ser mejores médicos e igualmente estar informados y aceptar que los enfermos puedan preguntar.

- Hemos de aprender a dar la información adecuada para cada uno de ellos, respetar sus tiempos, sus circunstancias y sus decisiones, aunque a veces sea complicado. Lo que nunca debemos hacer es mentir ni prometer cosas que no van a ocurrir.

- Es fundamental, como siempre, infundir confianza y que el paciente vea que no se le deja de lado, aunque no se sepa qué le ocurre.

- Sigo creyendo que el médico es parte fundamental del proceso curativo (...). Estoy convencido de que gastaríamos menos en salud si pudiéramos dedicarle un poco más de tiempo al paciente.

- ¿Cuál cree que es su principal papel como médico? Los médicos a veces podemos curar, otras veces sólo podemos aliviar, pero lo que podemos hacer siempre es acompañar a nuestros pacientes.

- Cuando tratas a los niños, primero hay que hacer que no tengan miedo, enseñarles, diciendo voy a hacer esto y después hacer que sonrían y confíen. Es importante no alejarles mucho de la madre.

- Siempre he tenido muy claro que debemos transmitir seguridad y confianza, tanto al paciente como a su familia (...), y a nuestro equipo de trabajo.

Tema 13. Psicología del enfermo en situaciones específicas

- Respecto al paciente hospitalizado y el protocolo de acogida a cargo del personal de enfermería (para distinguirlo de la expresión literal del profesional sanitario): de esta forma se le da sensación de confort y humanización desde el primer momento, se le intenta tranquilizar y se responde a sus primeras dudas e inquietudes, explicándoles también cómo funciona la unidad y asegurándoles la disponibilidad del equipo las 24 horas los 7 días de la semana.

- ¿Cuáles son las consecuencias psicológicas de los ingresos hospitalarios? ¿A qué se tiene más miedo y qué debe hacer el médico para minimizar las inquietudes del paciente?:

- Inquietud, ansiedad, preocupación, pensamiento de empeoramiento, separación de su ambiente familiar, abandono de su hogar, etc.

- Sensación de alivio e incluso tranquilidad. El hospital les da seguridad, sensación de estar más protegidos contra el cáncer, especialmente en situaciones de pacientes mayores, dependientes sin apoyo familiar, estados avanzados de la enfermedad, familias que gestionan mal la enfermedad en casa, etc.

- No son pocas las situaciones en que los pacientes y/o la familia rechazan el alta hospitalaria y es necesario contactar con cuidados paliativos, asistentes sociales, hospitales de larga estancia, etc.

Tema 16. El estrés

De los pacientes

- ¿Cómo lleva la gente las extensas listas de espera (de trasplante hepático)? Bueno, pues es una situación muy estresante. Cuando uno espera más de tres meses, realmente se pone nervioso. Los pacientes lo viven con mucha angustia.

- Cada paciente muestra (el estrés) de una manera; en ancianos ocurren muchas desorientaciones y confusiones, y otros pacientes lo demuestran con el decaimiento o con la ira. Se les nota que están ansiosos y preocupados.

- ¿Qué importancia tiene el estrés? Mucha, como precipitante, desencadenante y mantenedor de patología psiquiátrica, hay que enseñar a manejarlo y en esto la psicoterapia tiene una gran función.

- De la serenidad con la que un médico se enfrenta a una situación difícil depende el resultado de esa situación.

De los profesionales sanitarios

- Sí, el estrés es una constante en nuestra profesión y hay que lidiar con ello, lo cual se aprende.

- ¿Cómo maneja usted su propio estrés? Pues desconectando una vez que sales de la consulta, estando preparada y actualizada en conocimientos y compartiendo con otros compañeros de profesión. El trabajo en equipo es fundamental.

- Respecto a la pandemia por COVID-19: estamos todos cansados; no dormimos bien, malcomemos y nos llevamos a casa mucho dolor.

25,5 y desde que obtuvieron su especialidad de 18,9 (Tabla III).

Catorce de los médicos $(48,3 \%)$ procedían de la colaboración con el COMVA, nueve (31\%) ha- bían sido contactados por algún estudiante, tres $(10,3 \%)$ habían conocido la actividad en la misma universidad y dos $(6,9 \%)$, por un colega clínico (Tabla IV). En la tabla IV pueden consultarse también otros datos profesionales, como la especialidad de titulación, el tipo de centro de trabajo del que provenían y la universidad de titulación. En cuanto a la realización de la actividad, 17 participantes $(58,6 \%)$ emplearon la videoconferencia, $10(34,5 \%)$ realizaron la entrevista de forma telemática escrita y dos $(6,9 \%)$ por teléfono (Tabla IV).

\section{Calificación de la actividad entregada por los estudiantes}

Los estudiantes manifestaron, a través de sus coordinadores de grupo de trabajo y de los delegados de clase, su satisfacción con la actividad. Esto se vio reflejado en sus calificaciones finales, con notas todas ellas de notable o sobresaliente (media $=8,8$; desviación estándar $=0,65$; mínimo $=7,5$; máximo $=10)$.

\section{Satisfacción de los profesionales sanitarios}

En una escala de tipo Likert del 1 al 5 (de peor a mejor valoración), los profesionales participantes en la actividad valoraron la organización de la actividad con una puntuación media de 4,5, y la realización de la actividad por los estudiantes con 4,7 puntos. En la tabla V se muestran estos resultados desgranados por dimensiones.

\section{Deseo de los profesionales de justificación, retroalimentación y mantenimiento de su participación}

En cuanto a las preguntas prospectivas de cara a futuros cursos académicos, en el caso de que no hubiera restricciones a causa de la pandemia por COVID-19, 13 de los profesionales preferirían participar de forma presencial $(44,8 \%)$, seis de forma no presencial $(20,7 \%)$ y 10 de ellos $(34,5 \%)$ no tendrían preferencia al respecto. Asimismo, 24 (82,8\%) profesionales querrían ser contactados el próximo curso, dos $(6,9 \%)$ no querrían participar y tres $(10,3 \%)$ no respondieron a esta pregunta (Tabla V). Por último, 28 profesionales $(96,6 \%)$ solicitaron información sobre los resultados de la actividad y sólo uno $(3,4 \%)$ declinó recibirlos. Además, 27 clínicos $(93,1 \%)$ solicitaron un justificante de participación en la actividad, mientras que sólo dos $(6,9 \%)$ no lo desearon (Tabla V). 


\section{Discusión}

En la asignatura Psicología del grado en Medicina, a través de la actividad 'Entrevista a un profesional sanitario', se ha diseñado e implementado una metodología docente innovadora durante el segundo cuatrimestre del curso académico 2019-2020, que se ha desarrollado en su mayor parte en régimen de docencia no presencial a causa del confinamiento fortuito y obligatorio por la pandemia de COVID-19 iniciado en marzo de 2020. Se trata de una actividad grupal en la que, tal como se ha pormenorizado en el presente artículo, los estudiantes deben planificar, realizar y comentar una entrevista a un profesional de ciencias de la salud. Se ha obtenido un elevado grado de satisfacción en los estudiantes y profesionales participantes, tal como evidencian las calificaciones de los primeros y los resultados de la encuesta realizada por los segundos.

\section{Características de la actividad}

Como se ha indicado, los objetivos fundamentales de la actividad son contribuir al contacto temprano con la práctica asistencial y a la humanización de la medicina por medio de la experiencia de un profesional sanitario experimentado. Se pretende, pues, favorecer la adquisición por parte del estudiante de un conjunto de competencias, tanto transversales como específicas, establecidas por la Agencia Nacional de Evaluación de la Calidad y Acreditación en el Libro Blanco del Título de Grado en Medicina [1]. Entre las competencias transversales que debe alcanzar todo graduado universitario se encuentran dos competencias instrumentales (2, capacidad de organización y planificación; 3, comunicación oral y escrita en la lengua nativa) y cuatro personales (9, trabajo en equipo; 12 , habilidades en las relaciones interpersonales; 13 , reconocimiento a la diversidad y la multiculturalidad; 15 , compromiso ético) [1]. Las segundas, competencias del graduado en Medicina que habilitan para ejercer la profesión de médico, están recogidas tanto en el mencionado Libro Blanco [1] como en la Orden ECI/332/2008 del Ministerio de Educación y Ciencia [10]. Entre ellas se encuentran, fundamentalmente, las seis correspondientes a A) Valores profesionales, actitudes y comportamientos éticos (por ejemplo, 1. Reconocer los elementos esenciales de la profesión médica, incluyendo los principios éticos, las responsabilidades legales y el ejercicio profesional centrado en el paciente; 4 . Desarrollar la práctica profesional con respeto a
Tabla III. Edad, sexo y años desde su formación de las muestras de estudiantes y profesionales participantes.

\begin{tabular}{lcc}
\hline Edad, media (desviación estándar) & Estudiantes & $\begin{array}{c}\text { Profesionales } \\
\text { sanitarios }\end{array}$ \\
\hline Sexo (hombres:mujeres, \%) & $19,7(2,5)$ & $49,2(8,9)$ \\
\hline
\end{tabular}

Estudiantes: número de matriculaciones

\begin{tabular}{lcc}
\hline Primera $(n, \%)$ & $156(95,1 \%)$ & N/A \\
\hline Segunda $(n, \%)$ & $7(4,3 \%)$ & N/A \\
\hline Cuarta $(n, \%)$ & $1(0,6 \%)$ & N/A \\
\hline
\end{tabular}

Estudiantes: vía de acceso a los estudios

\begin{tabular}{lcc}
\hline Pruebas de acceso a la universidad $(n, \%)$ & $160(97,6 \%)$ & N/A \\
\hline Reconocimiento de estudios $(n, \%)$ & $2(1,2 \%)$ & N/A \\
\hline Programas internacionales $(n, \%)$ & $2(1,2 \%)$ & N/A
\end{tabular}

Profesionales sanitarios: años transcurridos desde...

\begin{tabular}{lll}
\hline Su titulación (media, desviación estándar) & N/A & $25,5(9,2)$ \\
\hline Su especialización (media, desviación estándar) & N/A & $18,9(9,2)$ \\
\hline
\end{tabular}

N/A: no aplicable.

la autonomía del paciente, a sus creencias y cultura), así como tres de D) Habilidades de comunicación (por ejemplo, 24. Establecer una buena comunicación interpersonal que capacite para dirigirse con eficiencia y empatía a los pacientes, los familiares, los medios de comunicación y otros profesionales). De forma adicional, pueden favorecerse otras competencias del médico: B) Fundamentos científicos de la medicina (como 9. Comprender y reconocer los efectos, mecanismos y manifestaciones de la enfermedad sobre la estructura y función del cuerpo humano, y 10. Comprender y reconocer los agentes causantes y factores de riesgo que determinan los estados de salud y el desarrollo de la enfermedad, en particular los de tipo psicológico y social); y E) Salud pública y sistemas de salud (aspectos psicológicos y sociales de 25. Reconocer los determinantes de salud en la población, tanto los genéticos como los dependientes del sexo y el estilo de vida, los demográficos, los ambientales, los so- 
Tabla IV. Información profesional de los sanitarios participantes y del modo de conocimiento y realización de la actividad.

\begin{tabular}{|c|c|c|c|c|c|}
\hline & $n$ & $\%$ & & $n$ & $\%$ \\
\hline Titulación & & & Especialidad de medicina & & \\
\hline Medicina & 26 & 89,7 & Familiar y comunitaria & 8 & 27,6 \\
\hline Enfermería & 3 & 10,3 & Psiquiatría & 4 & 13,8 \\
\hline Universidad de titulación & & & Intensiva & 3 & 10,3 \\
\hline Valladolid & 23 & 79,3 & Digestiva & 2 & 6,9 \\
\hline Salamanca & 2 & 6,9 & $\begin{array}{l}\text { Anestesiología } \\
\text { y reanimación }\end{array}$ & 2 & 6,9 \\
\hline Complutense & 2 & 6,9 & Endocrinología y nutrición & 1 & 3,4 \\
\hline Cantabria & 1 & 3,4 & Otorrinolaringología & 1 & 3,4 \\
\hline Oviedo & 1 & 3,4 & Inspección médica & 1 & 3,4 \\
\hline Centro de trabajo & & & Pediatría & 1 & 3,4 \\
\hline Hospital & 16 & 55,2 & Oncología radioterápica & 1 & 3,4 \\
\hline Centro de salud & 6 & 20,7 & Interna & 1 & 3,4 \\
\hline Emergencias & 3 & 10,3 & Preventiva y salud pública & 1 & 3,4 \\
\hline Consulta privada & 2 & 6,9 & Enfermería & 3 & 10,3 \\
\hline Inspección & 1 & 3,4 & Conocimiento de la actividad & & \\
\hline Gerencia & 1 & 3,4 & COM de Valladolid & 14 & 48,3 \\
\hline Realización de la actividad & & & Por un estudiante & 9 & 31 \\
\hline Videoconferencia & 17 & 58,6 & Universidad & 3 & 10,3 \\
\hline Escrita & 10 & 34,5 & Por un colega & 2 & 6,9 \\
\hline Teléfono & 2 & 6,9 & No contesta & 1 & 3,4 \\
\hline
\end{tabular}

COM: Colegio Oficial de Médicos. empleo de este tipo de aprendizaje en futuras actividades prácticas asistenciales incluidas en el currículo del grado en Medicina y en la formación del médico interno residente.

Es indudable que, durante su formación académica, los estudiantes tienen repetidas ocasiones de interacción con profesionales sanitarios de diversos entornos y especialidades, en particular durante la realización de prácticas clínicas, interacciones que son determinantes para el aprendizaje del estudiante [11]. Sin embargo, no hemos hallado ninguna publicación precedente que recoja la realización de entrevistas a profesionales como método docente establecido en la formación de graduados en Medicina o bien de alguna de las restantes profesiones sanitarias. Por el contrario, sí se han utilizado entrevistas en el ámbito educativo, tanto para la promoción de hábitos saludables en adolescentes de entornos desfavorecidos - como en nuestro caso, también a profesionales sanitarios- [12] o en estudiantes de Pedagogía, para crear un programa de radio, tipo podcast, a profesionales expertos en tecnologías de la información y la comunicación, y educación [13].

La actividad que se propone facilita el conocimiento y el contacto asistencial temprano, que constituye un elemento esencial para la comprensión de la actividad médica en estudiantes de los primeros cursos [14]. Entre otros beneficios, este contacto temprano podría incrementar la vocación médica, que ha de ser entendida como un proceso a lo largo de la formación académica de estudiantes de Medicina [15]. Los beneficios del contacto clínico y la práctica temprana en las formaciones sanitarias incluyen al aprendizaje y el desarrollo de actitudes y valores profesionales, especialmente vinculados al currículo oculto (serie de lecciones, valores y perspectivas no escritas de forma oficial en su programa de formación) que los profesionales clínicos pueden hacer visible a los futuros médicos [16].

La actividad que se presenta requiere la participación activa del estudiante -o grupo de estudiantes, en nuestro caso- en el diseño, la realización y la valoración del contenido de la entrevista. Permite, pues, una mayor implicación, y constituye por ello una 'metodología activa' o centrada en el aprendizaje del estudiante. Entre los diversos tipos de estas metodologías se encuentra el aprendizaje basado en proyectos, en el cual se proporciona a los estudiantes contextos de aprendizaje reales y se les implica en el diseño, en la resolución del problema y, sobre todo, en la toma de de- 
Tabla V. Izquierda: valoraciones dimensionales y totales de la organización y la actividad (escalas de tipo Likert de 1 a 5, de peor a mejor valoración). Derecha: resultados prospectivos para próximos cursos y deseo de retroalimentación.

\begin{tabular}{|c|c|c|c|c|c|c|c|c|c|}
\hline & $n$ & Media & DE & & $n$ & $\%$ & & $n$ & $\%$ \\
\hline \multicolumn{4}{|l|}{ Organización de la actividad: } & \multirow{2}{*}{\multicolumn{3}{|c|}{ ¿Desea ser contactado para el próximo curso? }} & \multirow{2}{*}{\multicolumn{3}{|c|}{ ¿Desea recibir un justificante de participación? }} \\
\hline Inscripción a la actividad & 29 & 4,3 & 0,7 & & & & & & \\
\hline Realización de la actividad & 28 & 4,6 & 0,6 & No & 2 & 6,9 & No & 2 & 6,9 \\
\hline Total & 28 & 4,5 & 0,5 & $\mathrm{NS} / \mathrm{NC}$ & 3 & 10,3 & $\mathrm{NS} / \mathrm{NC}$ & 0 & 0 \\
\hline
\end{tabular}

Actividad realizada por los estudiantes:

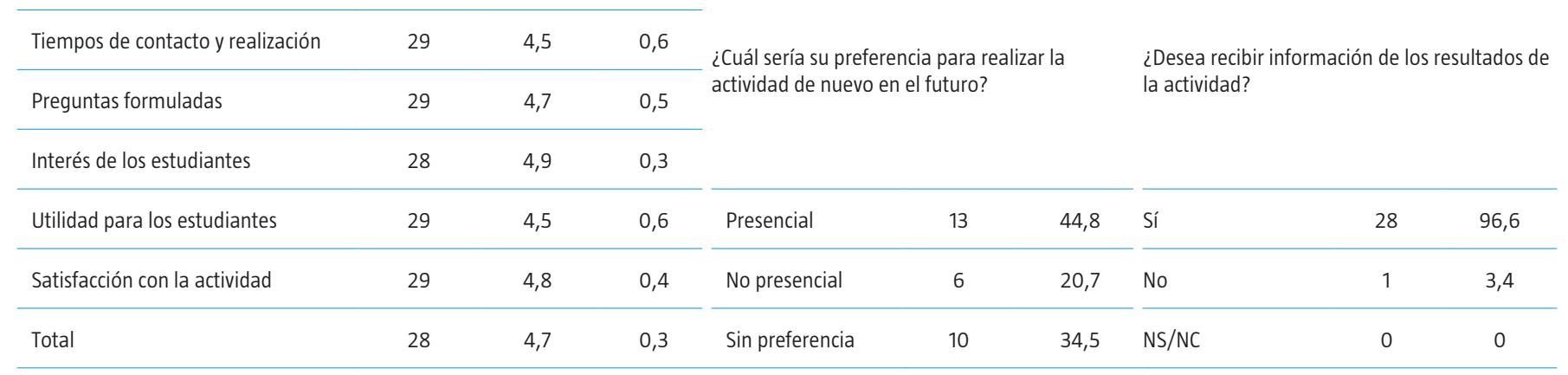

DE: desviación estándar; NS/NC: no sabe/no contesta.

cisiones [13]. Además de ello, y también como característica del aprendizaje basado en proyectos, la realización de la actividad se prolonga durante un espacio de tiempo y culmina -para el estudiante- con la entrega de un determinado resultado o 'entregable', en nuestro caso, resumen y comentario de la entrevista. Asimismo, nuestra actividad se realizó de forma grupal, lo que garantiza de este modo las bases del trabajo en equipo, fundamental en las ciencias de la salud y en especial en la medicina, para garantizar una labor eficiente y la seguridad de los pacientes [17].

\section{Satisfacción de los estudiantes y profesionales sanitarios}

La naturaleza de nuestra actividad se enmarca en los requerimientos del Espacio Europeo de Educación Superior, que se fundamenta, entre otros aspectos, en el aprendizaje permanente y el empleo del sistema de créditos ECTS, convirtiéndose los estudiantes en miembros activos de su propia educación y formación $[18,19]$. A pesar del esfuerzo que les supone, los estudiantes valoran de forma positiva su trabajo autónomo [20]. Evaluar la satisfacción de los estudiantes en una actividad docente es primordial para hacer también partícipe al alumnado y para que los docentes obtengamos retroalimentación que garantice su continuidad y mejora. El actual modelo educativo prevé que los estudiantes tengan la posibilidad de valorar y comentar la actividad docente de los profesores en las encuestas anuales que promueven las distintas universidades. A pesar de las dificultades impuestas por el confinamiento durante el curso 2019-2020, sobre un máximo de 5 puntos, las valoraciones a la actividad docente de los profesores de la asignatura de Psicología fueron muy elevadas (a la pregunta 'La asignatura ha resultado interesante', los estudiantes respondieron para cada profesor lo siguiente: N.J.: $n=62$, A.D.: $n=56$, media $=4,4$ sobre 5 y mediana $=5$ en ambos casos). Sin embargo, estas puntuaciones no reflejan de forma específica la valoración de los estudiantes a la actividad objeto de este artículo. Otra forma más específica de valorar la satisfacción en la actividad, aunque indirecta, es la calificación en ella 
[21]. En este sentido, los estudiantes obtuvieron en sus trabajos grupales una alta calificación $(8,8$ sobre 10 , notable alto), por encima de la calificación general media en la asignatura, por lo que podemos deducir una adecuada satisfacción con la actividad.

Por otro lado, son escasos los trabajos que valoran las respuestas de los profesionales participantes en una actividad docente, tal como hemos realizado en el presente trabajo. En ciencias de la salud, algunos estudios han evaluado la satisfacción del profesorado en cuanto a la implantación de metodologías dirigidas al Espacio Europeo de Educación Superior, con resultados poco satisfactorios [22]. Se hace, pues, necesario, tal como hemos hecho en el presente trabajo, la implantación y la evaluación de actividades innovadoras originales en este campo. Tal como se han mostrado, nuestros resultados han sido muy satisfactorios por parte también de los profesionales clínicos participantes (Tabla V), lo que contrasta con las diferencias obtenidas por categorías profesionales respecto a la actividad asistencial [23].

\section{Adaptación de la docencia a la situación de pandemia por COVID-19}

La obligatoriedad de docencia no presencial debida a la pandemia de COVID-19, y por consiguiente la adopción de medidas de confinamiento durante el curso 2019-2020, ha supuesto la sustitución de la entrevista presencial, como se había previsto, por su realización, en la mayor parte de los casos, con apoyo de las tecnologías de la información y la comunicación. Estas herramientas han permitido también mantener un estrecho contacto con los coordinadores de grupo para el seguimiento de la actividad y solventar rápidamente algunas incidencias, elemento igualmente clave para su finalización con éxito por parte de todos los grupos. Efectivamente, la pandemia ha permitido un gran avance en la aplicación de las tecnologías de la información y la comunicación a la formación universitaria de grado, por ejemplo, en la enseñanza y el seguimiento de actividades no presenciales [24]. Sin duda, esta situación ha puesto de manifiesto la necesidad de cambios en la formación médica, que han de incluir tanto los métodos docentes como los de evaluación [25], como se propone en nuestra innovadora actividad. En efecto, tenemos la obligación de impulsar la transformación de fondo de la educación médica y de los profesionales de ciencias de la salud $[3,26,27]$. Esta crisis debería servir a médicos, docentes y estudiantes de Medici- na para extraer conclusiones y estar mejor preparados para el futuro [28].

\section{Limitaciones y fortalezas}

Este trabajo cuenta con la principal limitación de no haber aplicado a los estudiantes participantes una encuesta de satisfacción específica de la actividad, en la que se hubieran podido valorar, entre otros, los mismos aspectos organizativos que en los profesionales sanitarios. Asimismo, no se diseñó inicialmente para su aplicación en un contexto de docencia virtual, lo que conllevó algunas modificaciones y falta de cumplimiento de los plazos inicialmente establecidos.

Por otra parte, cuenta con la fortaleza del diseño y la aplicación de una actividad original a un numeroso grupo de estudiantes. Además, se han realizado numerosas valoraciones que permiten cuantificar tanto la satisfacción de los profesionales participantes en los múltiples aspectos de la actividad como la disponibilidad a continuar participando en ella, incluso en un contexto inédito de docencia no presencial. Todo ello permitirá mejorar el diseño y la implementación de la actividad en futuros cursos académicos.

\section{Futuras líneas de trabajo}

Se sugiere: a) la evaluación de la actividad por parte de los estudiantes; b) el estudio pormenorizado de algunas variables que pudieran intervenir en el resultado obtenido, como las características del profesional sanitario o el modo de realización de la entrevista (presencial o mediante tecnologías de la información y la comunicación), y c) su aplicación a otras titulaciones de profesiones sanitarias.

\section{Ideas clave}

- La realización de entrevistas a profesionales sanitarios por parte de grupos de estudiantes es una metodología docente innovadora aplicable al grado en Medicina y eventualmente a otras titulaciones de profesiones sanitarias.

- Esta metodología facilita el contacto precoz de los estudiantes de Medicina con la práctica clínica de diversos profesionales sanitarios, captados con la colaboración del colegio oficial de médicos de la provincia.

- La actividad requiere la participación activa del estudiante, estimula el trabajo en grupo y constituye un aprendizaje por proyectos. 
- La entrevista a profesionales sanitarios ha sido positivamente aceptada por los estudiantes y altamente valorada por parte de los profesionales sanitarios participantes.

- Las tecnologías de la información y la comunicación han permitido adaptar la actividad a la situación de confinamiento derivada de la pandemia por COVID-19.

\section{Bibliografía}

1. Peinado JM, coord. Libro Blanco del Título de Grado en Medicina. Madrid: ANECA; 2005.

2. Morán J. Competencias del médico del siglo xxı. Un cambio necesario. Rev Cienc UP 2019; 1: 128-43.

3. Abreu-Hernández LF, León-Bórquez R, García-Gutiérrez JF. Pandemia de COVID-19 y educación médica en Latinoamérica. FEM 2020; 23: 237-42.

4. Albarrán Torres F, Urrutia Martínez M, Ibarra Peso J, Miranda Díaz C, Meza Vásquez S. Maquetas como estrategia didáctica en estudiantes de la salud. Educ Med 2020; 21: 198-206.

5. Guerra-Zúñiga M, Cuevas-Lang K, Segovia-Chamorro J. ¿Qué tecnología escojo para mi clase? Percepción de estudiantes de medicina sobre clickers y Kahoot. FEM 2020; 23: 111-5.

6. Benavides FG, Segura A. Una experiencia docente en el grado de medicina: 'Introducción al sistema de salud.' FEM 2016; 19: 115-7.

7. Romero-Palacios PJ, Romero-López MA, Gómez-Jiménez FJ, Miranda-León MT. Aprendizaje práctico de la medicina en entornos clínicos para alumnos de tercer curso: consecución de objetivos de conocimiento. Educ Med 2012; 15: 235-46.

8. Mejías-Vizcarro JA, Beltrán-Hernández L. Utilidad de la historia médico-psicológica en estudiantes que inician ciclos clínicos en la carrera de medicina como instrumento para la comprensión integral del paciente y facilitador de la alianza terapéutica. FEM 2019; 22: 27-34.

9. Millán J. Humanización de la medicina, medicina humanizada, medicina humanista: ¿de qué estamos hablando? Editorial. Educ Med 2018; 19: 131-2.

10. Ministerio de Educación y Ciencia. Orden ECI/332/2008, de 13 de febrero, por la que se establecen los requisitos para la verificación de los títulos universitarios oficiales que habiliten para el ejercicio de la profesión de médico. Boletín Oficial del Estado 40, de 15 de febrero de 2008. p. 8351-5.

11. García-Carpintero Blas E, Siles-González J, Martínez-Roche ME, Martínez-Miguel E, Manso-Perea C, González-
Cervantes S, et al. Percepciones de los estudiantes sobre sus vivencias en las prácticas clínicas. Enferm Univ 2019; 16: 259-68.

12. González F. Entrevista a un profesional sanitario. Ene Rev Enferm 2013; 7: 1-5.

13. Ausín V, Abella V, Delgado V, Hortigüela D. Aprendizaje basado en proyectos a través de las TIC. Una experiencia de innovación docente desde las aulas universitarias. Form Univ 2016; 9: 31-8.

14. Baños JE, Sentí M, Miralles R. Contacto precoz con la realidad asistencial: una experiencia piloto en medicina. Educ Med 2011; 14: 39-47.

15. Villaplana-Soto AE, Altisent R, Delgado-Marroquín MT. La vocación médica, ¿nace o se hace? Impacto del Grado de Medicina en la sensibilidad ética del alumno. FEM 2019; 22: 227-33.

16. Nolla Domenjó M. Aprendizaje y prácticas clínicas. Educ Med 2019; 20: 100-4.

17. Mujumdar S, Santos D. Teamwork and communication: an effective approach to patient safety. World Hosp Health Serv 2014; 50: 19-22.

18. García Martín J, Pérez Martínez JE. Aprendizaje basado en proyectos: método para el diseño de actividades. Rev Tecnol Cienc y Educ 2018; 9: 31-8.

19. Declaración de Bolonia. Declaración conjunta de los ministros europeos de educación reunidos en Bolonia el 19 de junio de 1999.

20. Rodríguez-Izquierdo RM. Modelo formativo en el Espacio Europeo de Educación Superior: valoraciones de los estudiantes. Aula Abierta 2014; 42: 106-13.

21. Hernández Vaquero L. Rendimiento, motivación y satisfacción académica, ¿una relación de tres? ReiDoCrea 2018; 7: 92-7.

22. Ariza T, Quevedo-Blasco R, Ramiro MT, Bermúdez MP. Satisfaction of health science teachers with the convergence process of the european higher education area. Int J Clin Heal Psychol 2013; 13: 197-206.

23. Carrillo-García C, Martínez-Roche ME, Gómez-García CI, Meseguer-De-Pedro M. Satisfacción laboral de los profesionales sanitarios de un hospital universitario: análisis general y categorías laborales. An Psicol 2015; 31: 645-50.

24. Palés-Argullós J, Gomar-Sancho C. Repercusiones de la COVID-19 en la educación de los médicos: la formación de grado (II). FEM 2020; 23: 161-6.

25. Gual A. Repercusión de la COVID-19 en la educación de los médicos: de la formación al aprendizaje (I). Editorial. FEM 2020; 23: 103-5.

26. Costa MJ, Carvalho-Filho M. A new age for medical education after COVID-19. FEM 2020; 23: 55-7.

27. Rose S. Medical student education in the time of COVID-19. JAMA 2020; 323: 2131-2.

28. Sacristán JA, Millán J. El médico frente a la COVID-19: lecciones de una pandemia. Educ Med 2020; 21: 265-71. 